\title{
Imaging Reveals Synaptic Targets of a Swim-Terminating Neuron in the Leech CNS
}

\author{
Adam L. Taylor, ${ }^{1,2}$ Garrison W. Cottrell, ${ }^{1}$ David Kleinfeld, ${ }^{3}$ and William B. Kristan Jr ${ }^{2}$ \\ ${ }^{1}$ Department of Computer Science and Engineering, ${ }^{2}$ Neurobiology Section, Division of Biological Sciences, and ${ }^{3}$ Department of Physics, University of \\ California, San Diego, La Jolla, California 92093
}

In the leech, the command-like neuron called cell $\operatorname{Tr} 2$ is known to stop swimming, but the connections from cell $\operatorname{Tr} 2$ to the swim central pattern generator have not been identified. We used fluorescence resonance energy transfer voltage-sensitive dyes to identify three neurons that are synaptic targets of cell Tr2. We then used electrophysiological techniques to show that these connections are monosynaptic, chemical, and excitatory. Two of the novel targets, cell 256 and cell 54, terminate swimming when stimulated. These neurons are likely to mediate swim cessation caused by cell Tr2 activity, and thus play the role of intermediate control cells in the leech CNS.

Key words: connectivity; leech; swimming; swim termination; voltage-sensitive dye; fluorescence resonance energy transfer; coherence; command neuron

\section{Introduction}

Hierarchical control is an organizational pattern found in both invertebrate and vertebrate nervous systems. In vertebrate nervous systems, the forebrain and brainstem are involved in the initiation, termination, and coordination of complex rhythmic behaviors, but the cycle-to-cycle control of these behaviors is mediated by pattern-generating networks in the spinal cord (Grillner et al., 1997). Similar hierarchical control is found in invertebrates, with higher-order neurons activating pattern generators, which then synapse on motor neurons (Orlovsky et al., 1999).

Single neurons that evoke or terminate whole behaviors (command-like neurons) have been found in many invertebrate preparations, whereas they seem to be rare in vertebrate preparations, presumably because of the larger number of neurons in these systems (Kupfermann and Weiss, 1978; Pearson, 1993; but see Roberts et al., 1997; Perrins et al., 2002). For instance, stimulating a single command-like neuron in the leech (Weeks and Kristan, 1978; Brodfuehrer and Friesen, 1986a) or in Tritonia (Frost and Katz, 1996) can elicit full-blown bouts of swimming, whereas it is necessary to stimulate many neurons in the brainstem of the lamprey (Orlovsky et al., 1999) to achieve a similar effect.

In the leech, in addition to swim-initiating neurons, there are command-like neurons that terminate an ongoing swim when

\footnotetext{
Received Sept. 1, 2003; revised 0ct. 8, 2003; accepted 0ct. 8, 2003.

This work was supported by a La Jolla Interfaces in Science Predoctoral Fellowship, funded by the Burroughs Wellcome Fund (A.L.T.); National Institutes of Health (NIH) Training Grant GM08107 (A.L.T.); and NIH Research Grants MH43396 (G.W.C., D.K., W.B.K.), RR13419 (D.K.), and NS35336 (W.B.K.). We thank J. E. Gonzalez and R. Y. Tsien for assistance with the FRET voltage-sensitive dyes; Panvera LLC for supplying the dyes gratis; K. Briggman, S. B. Mehta, T. M. Esch, the Kristan Laboratory, and GURU (Gary's Unbelievable Research Unit) for valuable discussions; and the two anonymous reviewers.

Correspondence should be addressed to Adam L. Taylor, Volen Center, Mail Stop 013, Brandeis University, 415 South Street, Waltham, MA 02454-9110. E-mail: altaylor@brandeis.edu.

Copyright $\odot 2003$ Society for Neuroscience $\quad$ 0270-6474/03/2311402-09\$15.00/0
}

stimulated (Brodfuehrer and Friesen 1986a; Brodfuehrer and Burns, 1995; O'Gara and Friesen 1995). One of these, cell Tr2, is found in the head brain of the leech (Brodfuehrer and Friesen 1986a; O'Gara and Friesen, 1995). Stimulation of this cell terminates an ongoing swim bout. It is not known, however, how cell $\operatorname{Tr} 2$ connects to the swim central pattern generator (CPG), and thus it is not known how it causes swim termination. One possibility is that cell $\operatorname{Tr} 2$ connects directly to the swim CPG cells. For instance, cell $\operatorname{Tr} 2$ could terminate swimming by inhibiting all or most of the swim CPG or by exciting part of the swim CPG in such a way as to disrupt rhythm generation. Such a scheme is used in the Xenopus tadpole, with mid-hindbrain reticulospinal neurons directly inhibiting the motor neurons involved in swimming (Perrins et al., 2002). A second possibility is that cell Tr2 inhibits the known swim-gating cells, thereby stopping swimming through these intermediaries. Because the swim-gating cells are necessary for the maintenance of swimming, this should be sufficient to stop an ongoing swim (Weeks, 1981; Nusbaum and Kristan, 1986). A third possibility is that cell $\operatorname{Tr} 2$ activates an intermediate network that then projects to the swim CPG cells and swim-gating cells. This intermediate network might be part of command-to-motor pathways for multiple behaviors, and together with the swim-gating cells could provide a neuronal locus in which pro-swimming and anti-swimming inputs are resolved. A precedent for this organization is provided by studies in which conflicting sensory inputs lead to a single, defined behavioral output (Kristan and Shaw, 1997).

As a first step toward resolving which of these schemes (if any) is used in the leech, we used fluorescence resonance energy transfer (FRET) voltage-sensitive dye imaging to search for synaptic targets of cell Tr2 (González and Tsien, 1995, 1997; Cacciatore et al., 1999; Zochowski et al., 2000). We found three neurons in the midbody ganglia that receive monosynaptic input from cell $\mathrm{Tr} 2$ and determined that two of these synaptic targets were able to terminate swimming when stimulated. None of these cells are 
either swim CPG cells or swim-gating cells, suggesting that they are part of an intermediate network by which Tr2 activity terminates swimming.

\section{Materials and Methods}

Preparation. Subjects were adult Hirudo medicinalis (4-8 gm), the European medicinal leech, obtained from Leeches USA (Westbury, NY) and maintained in artificial pond water at $15^{\circ} \mathrm{C}$. We dissected out the full nerve cord, including the head brain, all 21 midbody ganglia, and the tail brain. We removed the connective tissue sheath from ganglia in which we planned to impale cells or image activity. Usually we desheathed the ventral surface of the head brain (subesophageal ganglion only) and the ventral side of one of the midbody ganglia from segments $10-13$. We prepared at least one dorsal posterior (DP) nerve, usually chosen from ganglia 14 through 17, for extracellular recording. A motor neuron in this nerve bursts during the dorsal contractile phase of swimming, so the nerve serves as a monitor of swimming activity (Ort et al., 1974). In some experiments, we stabilized the imaged ganglion by pinning small strips of sausage casing across them to minimize motion artifact (Cacciatore et al., 1999). The preparation was maintained in a chamber filled with $\sim 5 \mathrm{ml}$ of leech saline, consisting of (in mM): $115 \mathrm{NaCl}, 4 \mathrm{KCl}, 1.8 \mathrm{CaCl}_{2}, 1.5 \mathrm{MgCl}_{2}$, 10 dextrose, 4.6 Tris maleate, and 5.4 Tris base, $\mathrm{pH}$ 7.4.

Staining with FRET dyes. We first stained the ganglion to be imaged with the FRET donor, $\mathrm{N}$-(6-chloro-7-hydroxycoumarin-3-carbonyl)dimyristoylphosphatidylethanolamine (CC2-DMPE) (Panvera LLC, Madison, WI), a coumarin-labeled phospholipid (González et al., 1999). We made a $30 \mu \mathrm{M}$ staining solution from $3 \mu \mathrm{l}$ of $5 \mathrm{~mm}$ CC2-DMPE in DMSO, $1 \mu \mathrm{l}$ of $20 \mathrm{mg} / \mathrm{ml}$ pluronic F-127 in DMSO (Molecular Probes, Eugene OR), and $500 \mu \mathrm{l}$ of saline. We pinned out the nerve cord in a Sylgard-coated dish, placed a small plastic cylinder over it, and then sealed it with petroleum jelly to make a watertight chamber. We then replaced the saline in the chamber with the staining solution and stained the ganglion for $30 \mathrm{~min}$. During staining, we constantly recirculated the staining solution using a peristaltic pump (model RP-1; Rainin, Oakland, CA). After $30 \mathrm{~min}$, the staining solution was taken off, and the tissue was washed several times with fresh saline.

We next stained the whole nerve cord with the FRET acceptor, bis(1,3diethyl-thiobarbiturate)-trimethine oxonol $\left[\operatorname{DiSBAC}_{2}(3)\right]$ (Panvera LLC), an oxonol dye (Tsien, 1976; González and Tsien, 1995). We made an $8 \mu \mathrm{M}$ staining solution from $8 \mu \mathrm{l}$ of $12 \mathrm{mM} \mathrm{DiSBAC}_{2}(3)$ in DMSO and $12 \mathrm{ml}$ saline. This solution was then bath-sonicated for at least $1 \mathrm{~min}$. We replaced the bathing solution with this solution and left it on for at least $30 \mathrm{~min}$. In many experiments, we left the oxonol solution on for as long as imaging was done, replacing it with fresh staining solution every few hours. After taking off the staining solution, we replaced it with fresh saline that was sometimes continuously perfused using a gravity perfusion system. Leaving the oxonol in the bath did not increase background fluorescence because its extinction coefficient is small at $405 \mathrm{~nm}$, the wavelength of the excitation light, and oxonol has 20 -fold less fluorescent yield in aqueous solution than in the membrane (Rink et al., 1980).

Electrophysiology and cell fills. We recorded intracellularly from cells using 40-60 M $\Omega$ glass microelectrodes filled with $1 \mathrm{~m}$ potassium acetate, using an Axoclamp 2A amplifier (Axon Instruments, Foster City, CA). We recorded extracellularly using suction electrodes and a four-channel differential amplifier (model 1600; A-M Systems, Sequim, WA). We digitized all electrical data at $1-2 \mathrm{kHz}$ using a 12-bit analog-to-digital (Ato-D) board (PCI-MIO-16E-4; National Instruments, Austin, TX) and custom LabVIEW (National Instruments) software.

We filled individual neurons using either tetramethylrhodamine dextran [3000 molecular weight (MW); Molecular Probes] or Alexa 488 dextran (10,000 MW; Molecular Probes). Microelectrodes were backfilled using solutions with concentrations of $50 \mathrm{mg} / \mathrm{ml}$ (rhodamine) or 25 $\mathrm{mg} / \mathrm{ml}$ (Alexa). Cells were then impaled, and dye was iontophoretically injected. Dye was allowed to diffuse for $\sim 1 \mathrm{hr}$, and tissue was fixed overnight, dehydrated, cleared, and mounted on a slide for viewing. Fills were imaged using a confocal microscope (1024ES; Bio-Rad, Hercules, CA). Dyes were excited by the $488 \mathrm{~nm}$ (Alexa) or $568 \mathrm{~nm}$ (rhodamine) emission line of a KrAr laser (60-WL-DZ; Bio-Rad). Images shown in
Figure 3 were edited manually to remove background fluorescence using Corel Photo-Paint (Corel Corporation, Ottawa, Ontario, Canada).

Optical recording. We acquired fluorescence images using an upright microscope (Axioskop 2FS; Zeiss). We usually used a $40 \times, 0.8$ numerical aperture (NA) water-immersion objective (Acroplan; Zeiss), but occasionally used a $20 \times, 0.5 \mathrm{NA}$ water-immersion objective (Acroplan; Zeiss) when a wider field of view was desired. For epi-illumination we used a tungsten halogen lamp (64625 HLX; Osram Sylvania, Danvers, MA) in a standard housing (HAL 100; Zeiss), powered by a low-ripple power supply (JQE 15-12M; Kepco, Flushing, NY). For all imaging, we used only the coumarin emission, because it provided brighter fluorescence and higher voltage sensitivity. The filter set consisted of a $405 \pm 15 \mathrm{~nm}$ bandpass excitation filter, a $430 \mathrm{~nm}$ dichroic mirror, and a $460 \pm 25 \mathrm{~nm}$ bandpass emission filter (Chroma Technology Corporation, Brattleboro, VT). We used a water-cooled CCD camera (MicroMax 512 BFT; Roper Scientific, Tucson, AZ) operated in frame-transfer mode to acquire the optical data, at a frame rate of $20 \mathrm{~Hz}$. The CCD chip in this camera has $512 \times 512$ pixels, but we normally binned at $4 \times 4$ pixels, yielding a $128 \times$ 128 image. The quantum efficiency of the camera at the coumarin emission peak was $80 \%$. The CCD chip was maintained at $-25^{\circ} \mathrm{C}$ during imaging. Imaging data were acquired using the software package WinView/32 (Roper Scientific, Trenton, NJ). We synchronized the optical and electrical recordings by feeding the frame timing signals emitted by the camera into the A-to-D board, along with all the electrophysiology signals.

The combination of CC2-DMPE and $\mathrm{DiSBAC}_{2}(3)$ yielded sensitivities in the range of $2-8 \% / 100 \mathrm{mV}$ for $1 \mathrm{~Hz}$ sinusoidal voltage signals with a 10 $\mathrm{mV}$ amplitude, centered around a baseline voltage of $-50 \mathrm{mV}$. Much of the variation in sensitivity seemed to be attributable to differences in soma size, with larger somata giving higher sensitivity.

Analysis. After acquiring the data, we analyzed them using Matlab (The Mathworks, Natick, MA). We outlined the images of individual somata manually using a custom-made graphic user interface. All pixels within each cellular outline were then averaged in each frame, yielding a raw fluorescence signal for each cell, which we denote by $F(t)$. The noise in these single-cell signals was normally shot-noise dominated. Raw fluorescence signals were usually corrupted by a slow downward drift attributable to bleaching, but this was eliminated by fitting it with a mathematical function and dividing it out of the signal. For each cell outline, bleaching was fit by computing a moving average of $F(t)$ with a Gaussian window $(\sigma=500 \mathrm{msec})$. This slow signal we denote by $F_{\text {slow }}(t)$. Because this slow signal also includes the DC component of $F(t)$, we have:

$$
\frac{\Delta F}{F}(t)=\frac{F(t)}{F_{\text {slow }}(t)}-1 .
$$

We estimated coherence using multi-taper methods (Thomson, 1982; Percival and Walden, 1993; Cacciatore et al., 1999). Only the differences from the procedures used in Cacciatore et al. (1999) will be described. For our coherence estimates, we fixed the frequency resolution, $\Delta f$, defined as the half-width of the spectral bands, at $\Delta f=2 / 3 \mathrm{~Hz}$, and the number of tapers was adjusted according to the duration of the data set. For the data presented here, the trial duration was always $T=9.5 \mathrm{sec}$; thus the number of tapers with good leakage properties was:

$$
K=\lfloor 2 T \Delta f\rfloor-1=11 .
$$

To calculate standard errors for the coherence estimates, we used the jackknife (Thomson and Chave, 1991). For the error in the coherence phase, we used the same procedure as described by Cacciatore et al. (1999). For the coherence magnitude, we used a slightly different procedure. After calculating the coherence magnitude estimate, $C$, and the take-away-one coherence magnitude estimates, $C_{i}$, the estimate and the take-away-one estimates were replaced with the transformed values given by $y=f(C)=\ln \left[C^{2} /\left(1-C^{2}\right)\right]$, as suggested in Thomson and Chave (1991). The SE of the transformed estimate is given by the jackknife expression:

$$
\sigma_{y}=\sqrt{\frac{N}{N-1} \sum_{i=1}^{N}\left(y_{i}-y_{\bullet}\right)^{2}}
$$


where $y_{\bullet}=\sum_{i=1}^{N} y_{i}$. Thus a one SE bar on $y$ would be the interval $(y-$ $\left.\sigma_{y}, y+\sigma_{y}\right)$. The error bar used for $C$ is the interval $\left(f^{-1}\left(y-\sigma_{y}\right), f^{-1}(y\right.$ $\left.+\sigma_{y}\right)$ ), or:

$$
\left(\sqrt{\frac{1}{1-e^{-\left(y-\sigma_{y}\right)}}}, \sqrt{\frac{1}{1-e^{-\left(y+\sigma_{y}\right)}}}\right) .
$$

Because the distribution of $y$ is roughly Gaussian, this interval provides an $\sim 68 \%$ confidence interval. This interval also has the advantage that it is guaranteed to be a subset of $[0,1]$, which is not the case if the above transformation is not used.

To test whether the coherence magnitude of a given cell was significantly greater than zero, i.e., larger than would be expected by chance from a signal with zero coherence, we compared the estimate of $C$ with the null distribution for coherence magnitude. It can be shown that for the null distribution the coherence magnitude will exceed the value $\sqrt{1-\alpha^{1 /(K-1)}}$ only in $100 \alpha \%$ of trials (Hannan, 1970; Jarvis and Mitra, 2001). We used a low $\alpha$ level of 0.001 in all experiments, to avoid false positives. We also calculated the multiple comparisons $\alpha$ level for each trial, given by $\alpha_{\text {multi }}=1-(1-\alpha)^{n}$, where $n$ is the number of cells, and verified that it did not exceed 0.05 on any trial.

When plotting the coherence in polar plots (see Figs. $1 E, H, 2 B$ ), we corrected for the phase shift caused by the slow response of the dye signal by shifting the coherence phase by $+54^{\circ}$. This is the phase angle by which the fluorescence signal lags the voltage when the voltage trajectory is a 1 Hz sinusoid. It is close to $\tan ^{-1}(2 \pi \tau f)=66^{\circ}$, the theoretical phase lag for $f=1 \mathrm{~Hz}$, given the reported time constant of the dye, $\tau=360 \mathrm{msec}$ (Cacciatore et al., 1999). (The difference arises because the reported time constant was based on data for a range of frequencies, and the $+54^{\circ} \mathrm{shift}$ was based solely on data for $1 \mathrm{~Hz}$ signals.) The phase shift was not adjusted in any way on a per-trial basis to achieve a better match between coherence estimates for optical versus electrical signals.

Cell identification. We identified the novel neurons described here (cells 252,256, and the pair of cells 54) on the basis of their soma position, soma size, amplitude of action potential as recorded in the soma, and presence of one-for-one EPSPs from cell Tr2. All three cells are found on the ventral aspect of the ganglion. Cells 256 and 252 are found in the posterior medial packet, and cells 54 are found in the posterior lateral packets. In a few cases, the identity of a cell was established purely on the basis of soma position, size, and strong optical coherence with cell $\operatorname{Tr} 2$ at a phase lag between $60^{\circ}$ and $120^{\circ}$. This was done only after a cell had been singled out optically and verified electrically in many animals and only in unambiguous cases. Cells that were reliably identified in many animals were assigned numeric "names" (e.g., cell 252) by matching up their usual soma position and size with those on the canonical map of the leech ganglion (Muller et al., 1981).

Cell Tr2 was identified on the basis of the description given in Brodfuehrer and Friesen (1986a). Cell Tr1 was typically found just medial to the Retzius cell in segment R1, and cell Tr2 was typically just medial to cell $\operatorname{Tr} 1$. The soma of cell $\operatorname{Tr} 2$ was typically slightly larger than that of $\operatorname{Tr} 1$ $(\sim 60$ vs $\sim 50 \mu \mathrm{m})$, and both cells displayed large $(20-40 \mathrm{mV})$ action potentials that rose without prepotential. In all preparations in which we could elicit swimming (67 of 96), we verified that cell $\operatorname{Tr} 2$ stopped or strongly slowed swimming when stimulated. In all such cases but one, the cell initially identified as cell $\operatorname{Tr} 2$ on the basis of position, soma size, and spike shape was found to stop or strongly slow swimming. In seven preparations, we filled cell $\operatorname{Tr} 2$ with fluorescent tracer and verified that the morphology of the cell was consistent with that described in Brodfuehrer and Friesen (1986a).

\section{Results}

Using imaging, we identified three putative synaptic targets of cell $\operatorname{Tr} 2$ in the segmental ganglia

To identify candidate synaptic targets of cell Tr2, we imaged many neurons simultaneously while stimulating cell Tr2. A midbody ganglion (usually ganglion 10) was stained with both components of the FRET system (see Materials and Methods), and one cell Tr2 was impaled and stimulated via current injection (Fig. 1A). In each trial, we injected a series of pulse trains into cell $\operatorname{Tr} 2$, using a level of current sufficient to elicit one spike per pulse. Each train was $500 \mathrm{msec}$ long, with an intertrain interval of 500 msec, yielding a train frequency of $1 \mathrm{~Hz}$. Within each train, we used a pulse frequency of $20 \mathrm{~Hz}$. During stimulation, we imaged a region of the stained ganglion using a CCD camera, producing a time series of fluorescence images (Fig. $1 B$ ). We typically viewed approximately one-third of the ganglionic surface $(20-80$ cells) at a time. For each trial, we manually drew ellipses around each of the visible cell bodies (Fig. 1C) and assigned an alphanumeric label to each ellipse. We then averaged the pixel values inside the ellipse of each cell to provide a single time-varying optical signal for that cell (a subset of these signals is shown in Fig. 1D).

We quantified how strongly each cell responded to cell $\operatorname{Tr} 2$ activity by estimating the coherence between each optical signal and the electrical recording of the cell $\operatorname{Tr} 2$ membrane potential (Cacciatore et al., 1999) (see Materials and Methods). The coherence magnitude gives a measure of how well two signals correlate with one another at each frequency, with a value of 1 implying perfect correlation and a value of zero implying no correlation. The coherence phase gives the phase difference between the two signals at each frequency. We focused on the coherence at $1 \mathrm{~Hz}$, the stimulation frequency, where most of the power in the cell $\operatorname{Tr} 2$ voltage signal is concentrated.

We used the coherence magnitude at $1 \mathrm{~Hz}$ to rank the optical signals in order of how strongly they responded to Tr2 stimulation. The six optical signals shown in Figure $1 D$ are those that had the highest coherence magnitude at $1 \mathrm{~Hz}$. They are shown in descending order of magnitude. Because the coherence estimate is a statistic, we could calculate the coherence magnitude required to reach statistical significance. Only the top three signals in Figure $1 D$ (shown in color) responded strongly enough to conclude that their coherence with cell $\operatorname{Tr} 2$ was not attributable to chance. We refer to these cells as "followers" of cell Tr2, after Peterlin et al. (2000). These cells were considered to be good candidates for being synaptic targets of cell $\operatorname{Tr} 2$. The coherence phase at the stimulation frequency served as a measure of the latency of the response of a neuron to cell $\operatorname{Tr} 2$ stimulation (Fig. $1 E$ ). The phase can also distinguish excitatory from inhibitory effects (Cacciatore et al., 1999).

Using optical recording, we identified three followers of cell $\operatorname{Tr} 2$ that appeared in similar positions across several preparations. The most coherent of these was an unpaired cell in the posterior medial packet, cell 256. A cell in the approximate position of cell 256, which had strong coherence with cell $\operatorname{Tr} 2$, was seen in 45 preparations (Figs. $1 C-E$ ). The optical signal of cell 256 often displayed a near-sinusoidal modulation at the stimulation frequency. A second strongly coherent cell, cell 252, was unpaired and located in the same packet. A highly coherent cell in the approximate position of cell 252 was seen in 21 preparations (Figs. $1 C-E$ ). The most coherent cell in the posterior medial packet was usually in cell 256 position, with a cell in cell 252 position the next most coherent. A third strongly coherent cell was also identified, cell 54 (Figs. $1 F-H$ ). Unlike the others, it was a paired cell, with one homolog in each of the posterior lateral packets. We observed a strongly coherent cell 30 times, in 22 preparations, in the approximate position of cell 54. In addition to displaying large coherence magnitude, all four neurons typically had coherence phase lags between $60^{\circ}$ and $120^{\circ}$ (after correcting for the dye time constant; see Materials and Methods). Because the coherence is evaluated at $1 \mathrm{~Hz}$, this corresponds to a 
A
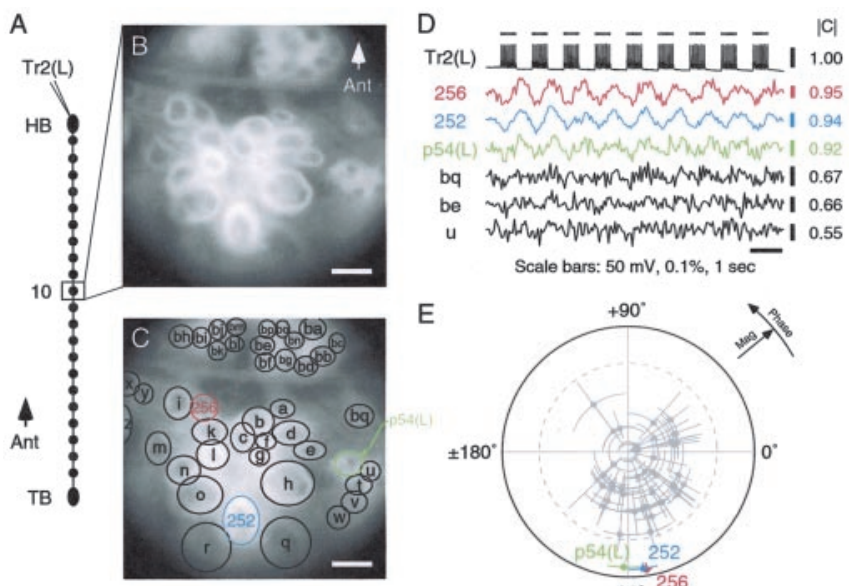

E
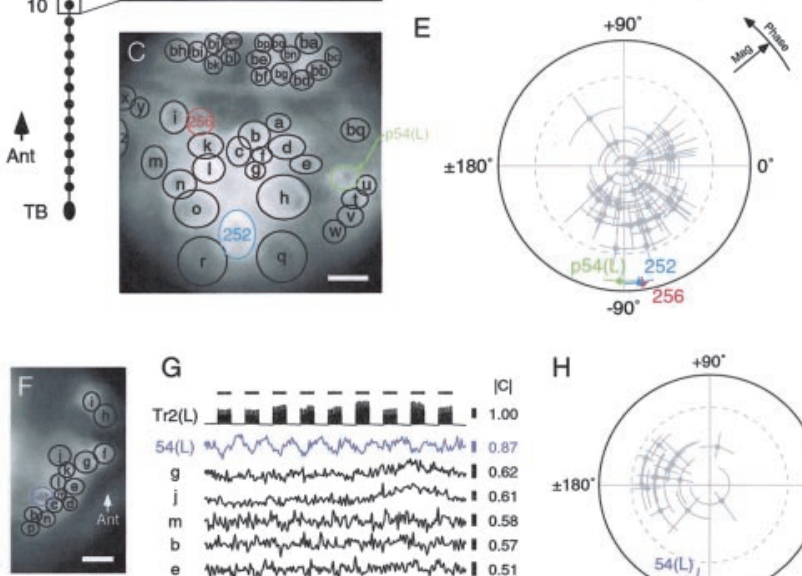

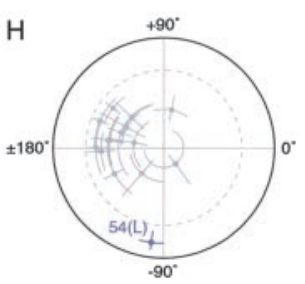

Figure 1. Optical discovery of cell $\operatorname{Tr} 2$ targets. A, A schematic of the preparation used to find cell Tr2 targets. The drawing represents the isolated leech nerve cord, consisting of the head brain (HB), 21 midbody ganglia (circles), and the tail brain (TB). Cell Tr2 was recorded intracellularly in the head brain, and the voltage-sensitive dye components were applied to a midbody ganglion, in this case ganglion 10. Ant, Anterior. $B$, The raw fluorescence image of ganglion 10 , obtained by averaging all frames from a movie of cellular fluorescence over time. The frame includes the posterior medial packet and parts of the right and left posterior lateral packets, all of which are on the ventral side of the animal. Scale bar, $50 \mu \mathrm{m}$. C, Ellipses that were drawn by hand and used to average the pixels for each cell in the field. Each cell was given an arbitrary alphanumeric label unless it was impaled and definitively identified in that preparation. Cell p54(L) is likely to be cell $54(\mathrm{~L})$ (p is for putative), but we did not impale it and verify its identity in this preparation. Colored cells were significantly coherent with the cell $\operatorname{Tr} 2(\mathrm{~L})$ electrical recording, at the $\alpha=0.001$ level (see Materials and Methods). Cells in black were not significantly coherent. Colors were chosen only to match up cells in ( with traces in $D$ and points in $E$; they are otherwise arbitrary. D, Simultaneous electrical recording of cell $\operatorname{Tr} 2(\mathrm{~L})$ and optical recordings from six of the cells shown in C. An increase in fluorescence reflects a depolarization of the cell. The optical traces are ordered by the magnitude of their coherence with the cell $\operatorname{Tr} 2(\mathrm{~L})$ electrical recording at $1 \mathrm{~Hz}$. The coherence magnitude values are given to the right of each trace. The bars above the cell $\operatorname{Tr} 2(\mathrm{~L})$ voltage trace show when current was being passed into cell $\operatorname{Tr} 2(\mathrm{~L})$. E, Polar plot of the coherence between each optical recording and the cell Tr2 electrical recording, at the $1 \mathrm{~Hz}$ drive frequency, for the 43 cells circled in C. The distance from the center represents the coherence magnitude, and the angle represents the coherence phase. The outer circle represents a coherence magnitude of 1, the highest possible value. The dashed line indicates the $\alpha=0.001$ threshold for significance. The error bars represent 1SE. A positive-negative phase angle means the signal leads or lags the cell Tr2 signal by that amount. All coherence estimates for fluorescence signals are shifted by $+54^{\circ}$, to correct for the phase shift caused by the time constant of the dye response (see Materials and Methods). The data shown in $A-E$ can also be presented as a movie, which is available at www.jneurosci.org. $F$, Analogous to $C$, but for a preparation in which cell $54(L)$ was impaled to identify it definitively. The circled cells are in the left posterior packet of the ventral side. Cells in the posterior medial packet cannot be seen clearly because they are above the focal plane. Scale bar, $50 \mu \mathrm{m}$. $G, H$, Analogous to $D$ and $E$, but for the field shown in $F$.

time lag of between 167 and 333 msec. Given that a typical conduction velocity for a Tr2 spike is $\sim 15$ msec per segment (Brodfuehrer and Friesen, 1986a; our data not shown) and that these recordings were done in ganglion 10 , these latencies suggest a direct excitatory connection from cell $\operatorname{Tr} 2$ (Fig. 1 E, $H$ ).

\section{Cell $\operatorname{Tr} 2$ is monosynaptically connected to candidate targets}

After we found a strong follower of cell Tr2 using optical recording, we impaled the follower (in the same preparation) to determine the nature of its connection to cell Tr2. For all three cell types described above, we found that the strong coherence with cell $\operatorname{Tr} 2$ activity was attributable to summating volleys of EPSPs in the target cell [Fig. 2A (data for cell 252 is shown)]. As expected, the optical signal was a low-pass-filtered version of the electrical signal (because of the time constant of the dye), with a lower signal-to-noise ratio and a lower sampling rate. The strong agreement between the optical and electrical recordings produced a similarly strong agreement between the coherence estimates derived from the two recordings (Fig. $2 \mathrm{~B}$ ): the two estimates are within the error bars of one another.

When individual cell Tr2 spikes were elicited, they produced single EPSPs in all three cell types, at a constant latency, typically between 125 and $175 \mathrm{msec}$ (Fig. 2C). The latency seen is consistent with the conduction delay for the cell $\operatorname{Tr} 2$ spikes to travel from the head brain to ganglion 10 (Brodfuehrer and Friesen, 1986a). We checked for EPSPs in 30 of the 45 optically identified cells 256, in 4 of the 21 cells 252 , and in 6 of the 30 cells 54 . In every case, we found that single cell $\operatorname{Tr} 2$ spikes caused one-for-one EPSPs in the observed follower (Fig. 3A-C). To test whether these connections were monosynaptic, we changed the concentration of divalent cations in the saline and examined the responses of the three cell $\operatorname{Tr} 2$ followers (Figs. 3A-C) (Nicholls and Purves, 1970; Berry and Pentreath 1976; Byrne et al., 1978; Liao and Walters, 2002). In all cases, the EPSP was preserved in $20 \mathrm{mM} \mathrm{Ca}^{2+} / 20$ $\mathrm{mM} \mathrm{Mg}^{2+}$ saline, which blocks polysynaptic PSPs, and disappeared in $0 \mathrm{~mm}$ $\mathrm{Ca}^{2+} / 20 \mathrm{mM} \mathrm{Mg}^{2+}$ saline, which blocks chemical synaptic transmission. Thus the connections from cell $\operatorname{Tr} 2$ to all three neurons appear to be monosynaptic and chemically mediated.

For all cells, the latency of the EPSPs increased when the preparation was in 20 $\mathrm{mM} \mathrm{Ca}{ }^{2+} / 20 \mathrm{mM} \mathrm{Mg}^{2+}$ saline. This was presumably caused by a reduction in the conduction velocity of action potentials attributable to elevated spike threshold. We verified that conduction velocity was slower in this saline by recording en passant from the connective between ganglia 8 and 9 . We found that the spikes did indeed take longer to propagate to the recording site in $20 \mathrm{mM} \mathrm{Ca}^{2+} / 20 \mathrm{mM} \mathrm{Mg}^{2+}$ saline and that the increased EPSP latency scaled with the increased delay (data not shown).

\section{Morphology and segmental extent of cell Tr2 targets}

The three cell Tr2 targets had distinct morphologies, which we visualized by filling the cells with fluorescent tracers (Fig. 3D-F). Cell 256 (Fig. 3D) is an unpaired cell, with a roughly symmetric pattern of neuritic branching. It sends a single process into the anterior medial connective. Cell 252 (Fig. 3E) is also unpaired, with a roughly symmetric pattern of neuritic branches. Unlike cell 256, it sends two processes out of the ganglion, one in the anterior medial connective and one in the posterior medial connective. Cell 54 (Fig. $3 F$ ) is a paired cell. Each member of the pair has a large tuft of neurites ipsilateral to the soma and sends its primary neurite contralateral. The primary neurite divides into 

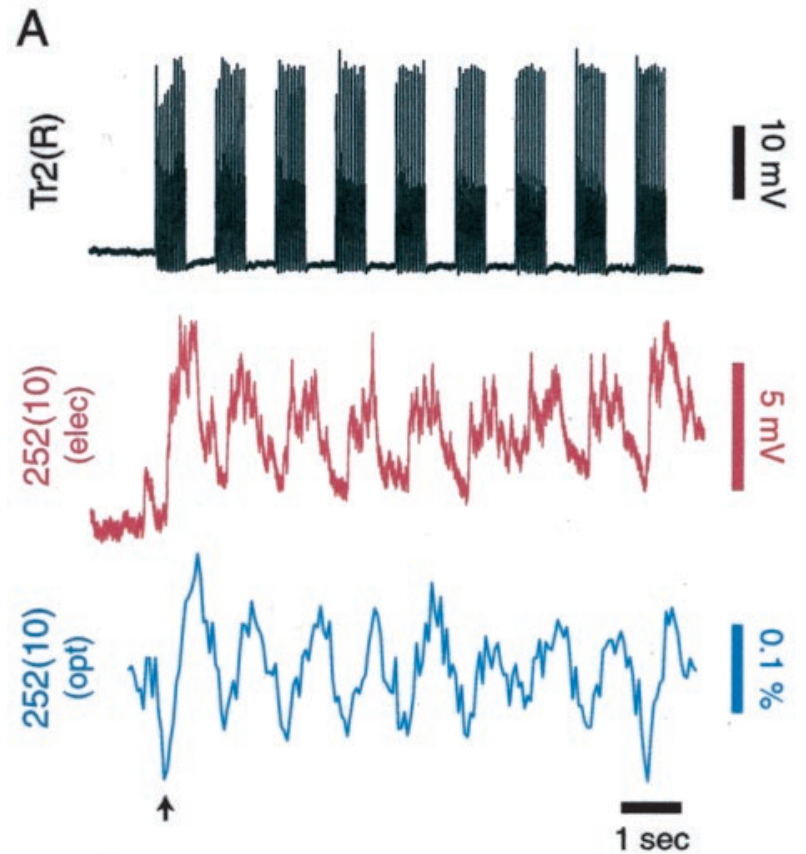

B

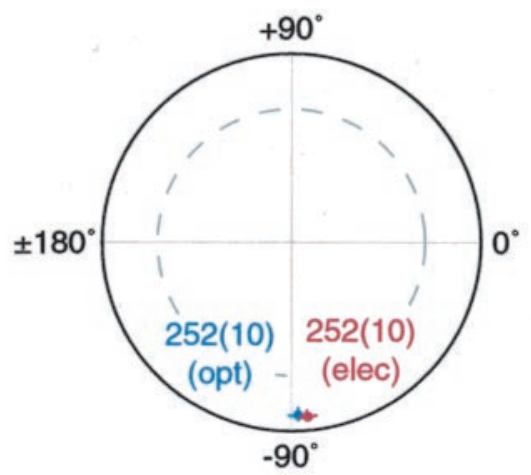

C
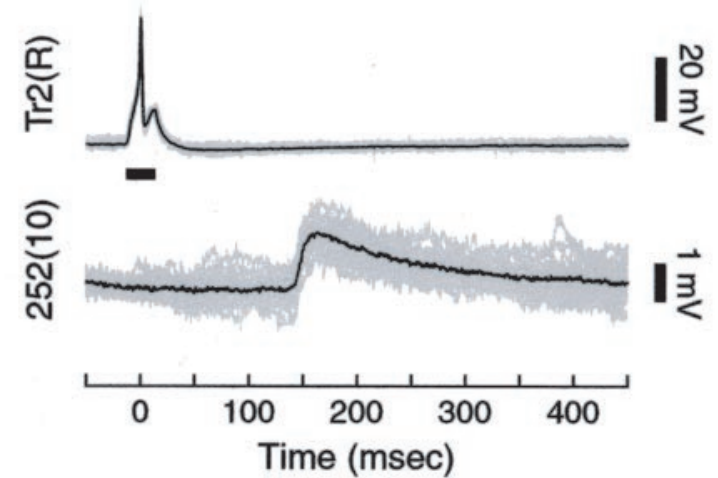

Figure 2. Simultaneous electrical and optical recordings from a cell Tr2 target, cell 252. A, Simultaneously recordings of cell Tr2(R) voltage, cell 252(10) voltage, and cell 252(10) fluorescence. Note the clear $1 \mathrm{~Hz}$ component in the fluorescence signal, despite the small size of the membrane potential fluctuations. The first negative deflection of the cell 252(10) optical recording (arrow) seems to precede the stimulus onset because of the filtering used to debleach the optical signals (see Materials and Methods). B, Coherence of the optical and electrical recordings of cell 252(10), both with respect to the cell $\operatorname{Tr} 2$ (R) membrane potential. As in Figure $1 E$, the coherence from the optical recording is shifted by $+54^{\circ}$ to correct for the phase shift caused by the time constant of the dye response (see Materials and Methods). The coherence for the electrical recording is not corrected. C, Activity in cells $\operatorname{Tr} 2(\mathrm{R})$ and $252(10)$, triggered on cell
A
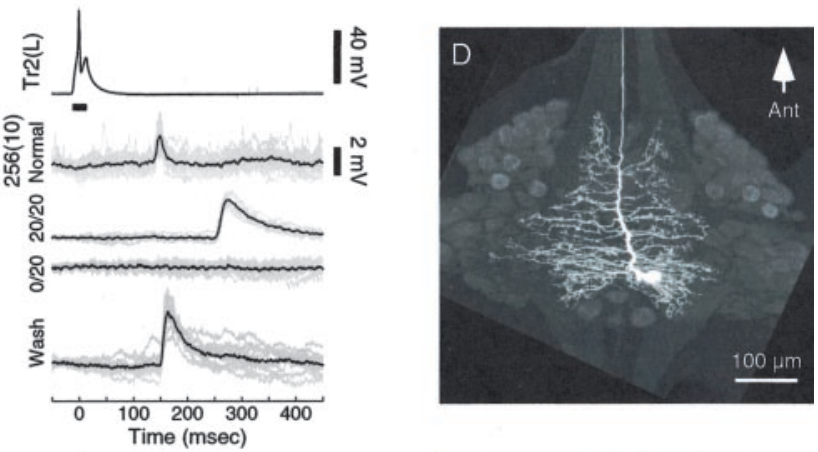

B
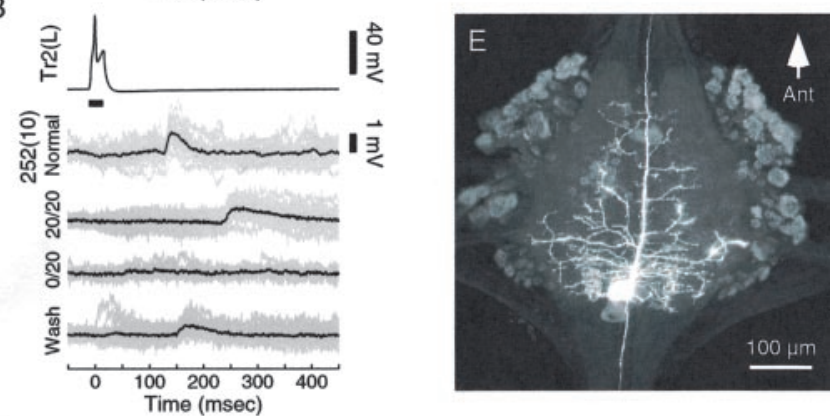

C
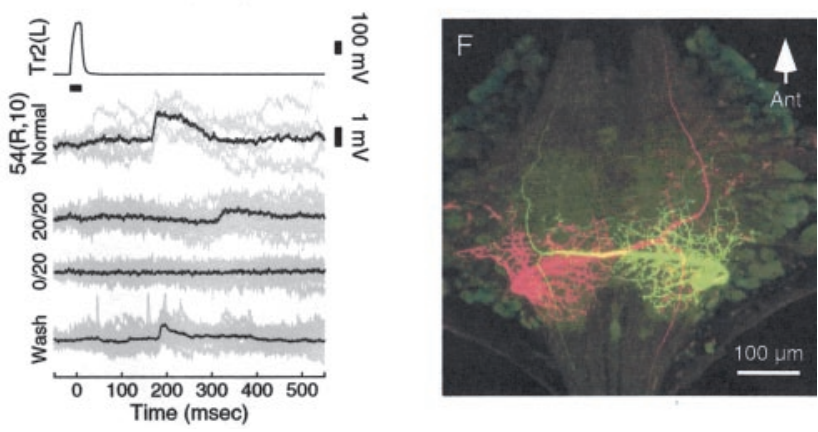

Figure 3. Monosynapticity and morphology of cell $\operatorname{Tr} 2$ targets. A, A series of spike-triggered averages (STAs) of simultaneous recordings from cells $\operatorname{Tr} 2(\mathrm{~L})$ and 256(10). In each case, the black trace shows the average of a number of individual sweeps, each of which is shown in gray. Four STAs, each from a different condition, are shown. The cell Tr2 spike that we used to trigger each sweep is shown only in the first condition for clarity of presentation. The cell Tr2 spike in normal saline is shown in the top trace. The black bar under the cell Tr2 trace indicates when current was passed. The second trace (Normal) is the simultaneously recorded activity in cell 256. The third trace (20/20) is the cell 256 recording from an STA done in $20 \mathrm{~mm} \mathrm{Ca}^{2+} / 20 \mathrm{~mm}$ $\mathrm{Mg}^{2+}$ saline. The fourth trace $(0 / 20)$ is in $0 \mathrm{~mm} \mathrm{Ca}{ }^{2+} / 20 \mathrm{~mm} \mathrm{Mg}^{2+}$ saline. The bottom trace (Wash) is again in normal saline. The four different salines were applied in that order, in a single preparation. Number of sweeps per condition are as follows: normal, 19; 20/20, 11; 0/20, 11; Wash, 11. $B$, Analogous data to that shown in $A$ but for cell 252 . Number of sweeps per condition are as follows: normal, 21;20/20,21;0/20,11; Wash, 21. C, Analogous data to that shown in $A$, but for cell 54. Number of sweeps per condition are as follows: normal, 6;20/20, 20;0/20, 20; Wash, 20. D, Confocal image of cell 256 filled with the fluorescent dye tetramethylrhodamine dextran. $E$, Confocal image of cell 252 filled with the fluorescent dye Alexa 488. F, Confocal image of cell $54(\mathrm{R})$ and $54(\mathrm{~L})$, filled with the fluorescent dyes tetramethylrhodamine dextran and Alexa 488, respectively.

two processes that then exit the ganglion, one via the contralateral (to the soma) anterior lateral connective and the other via the contralateral posterior lateral connective.

We found segmental homologs of cells 256, 252, and 54 in all

$\leftarrow$

Tr2 spikes. The black trace is a spike-triggered average of 21 individual sweeps, with the individual sweeps shown in gray. Each cell Tr2 spike is followed by an EPSP in cell 252, with a latency of $147 \pm 3$ msec (mean \pm SD). The black bar under the cell Tr2 trace indicates when current was passed. opt, Optical; elec, electrical. 
midbody ganglia investigated (segments 9 through 12). Using imaging or electrical recordings, or both, we identified a total of 36 cells $256(10)$ in as many animals, 20 cells 252(10) in as many animals, and 36 cells 54(10) in 24 animals. The homologs in other segments were identified one to four times each per segment examined. Given the extensive segmental homology of the leech body plan, it is likely that cells 256,252, and 54 are present in all or most midbody ganglia (Muller et al., 1981).

We found that both cell Tr2 homologs connected to all targets within a given midbody ganglion. That is, cells $\operatorname{Tr} 2(\mathrm{~L})$ and $\operatorname{Tr} 2(\mathrm{R})$ both connected to cells 256(n), 252(n), 54(L,n), and 54(R,n), where $n$ is the segment number (data not shown).

\section{Cells 256 and 54 terminate swimming}

We tested the ability of cells 256,252 , and 54 to terminate swimming by activating them individually during ongoing swimming episodes. We found that cells 256 and 54 stopped swimming episodes when driven strongly (Fig. 4) (data shown for cell 256 only). Consistent with this, cells 256 and 54 showed an increased firing rate during swim stops caused by cell $\operatorname{Tr} 2$ stimulation.

We found that driving cell 256 stopped swimming at least once in $67 \%$ of animals in which it was attempted $(n=9)$ (Fig. $4 A$ ) and that such stimulation stopped swimming in $50 \%$ of attempts $(n=26)$. We typically drove cell 256 at $\sim 30 \mathrm{~Hz}$ for 3-4 sec to obtain effective swim termination. This rate is substantially higher than the rate at which cell 256 spikes during cell Tr2induced swim stops. In the example shown in Figure $4 B$, cell 256 spiked at $7.3 \mathrm{~Hz}$ during a $\operatorname{Tr} 2$-induced swim stop $(n=1)$.

To verify that the apparent effect of cell 256 was not attributable to chance, we performed a more controlled experiment on two nerve cords. Each trial in this experiment consisted of a nerve shock, which initiated swimming, followed at a set latency by stimulation of cell 256 at a high rate for $4 \mathrm{sec}$. A trial was counted as a "success" if swimming stopped during the stimulus and a "failure" if it stopped after the stimulus. (We discarded trials in which swimming stopped before the onset of stimulation.) We then compared the success rate in this condition with the success rate in a control condition in which cell 256 was not stimulated. A control trial was counted as a success if the swim stopped during the "stimulation window," although no stimulus was delivered during this time. Swimming stopped during the stimulation window in $100 \%$ of the six stimulated trials, as compared with $20 \%$ of the five control trials. This difference is significant $(p<0.02$; one-sided Fisher-Irwin test).

A single cell 54 was also capable of stopping swimming when stimulated strongly, although cells 54 stopped swimming more effectively when both homologs were stimulated simultaneously (data not shown). In some trials, swimming slowed (burst frequency decreased) for the duration of cell 54 stimulation but did not stop. Driving both cells 54 slowed or stopped swimming at least once in $100 \%$ of animals $(n=5)$ and in $93 \%$ of trials overall $(n=14)$. Driving both cells 54 stopped swimming in $60 \%$ of individuals and in $29 \%$ of trials. Driving a single cell 54 slowed or stopped swimming at least once in $100 \%$ of animals $(n=4)$ and in $73 \%$ of trials overall $(n=22)$. Driving a single cell 54 stopped swimming in $50 \%$ of individuals in $9 \%$ of trials.

As with cell 256, the spike rates needed to stop swimming by driving cells 54 were higher than the rates observed when we stopped swimming by stimulating cell $\operatorname{Tr} 2$. The average spike rate required for cell 54 to stop swimming was $42 \pm 11 \mathrm{~Hz}(n=5$; in three animals), whereas its average spike rate when a cell $\operatorname{Tr} 2$ stopped swimming was only $2.8 \pm 0.9 \mathrm{~Hz}(n=3$; in two animals).

The role of cell 252 in terminating swimming is not clear. Cell
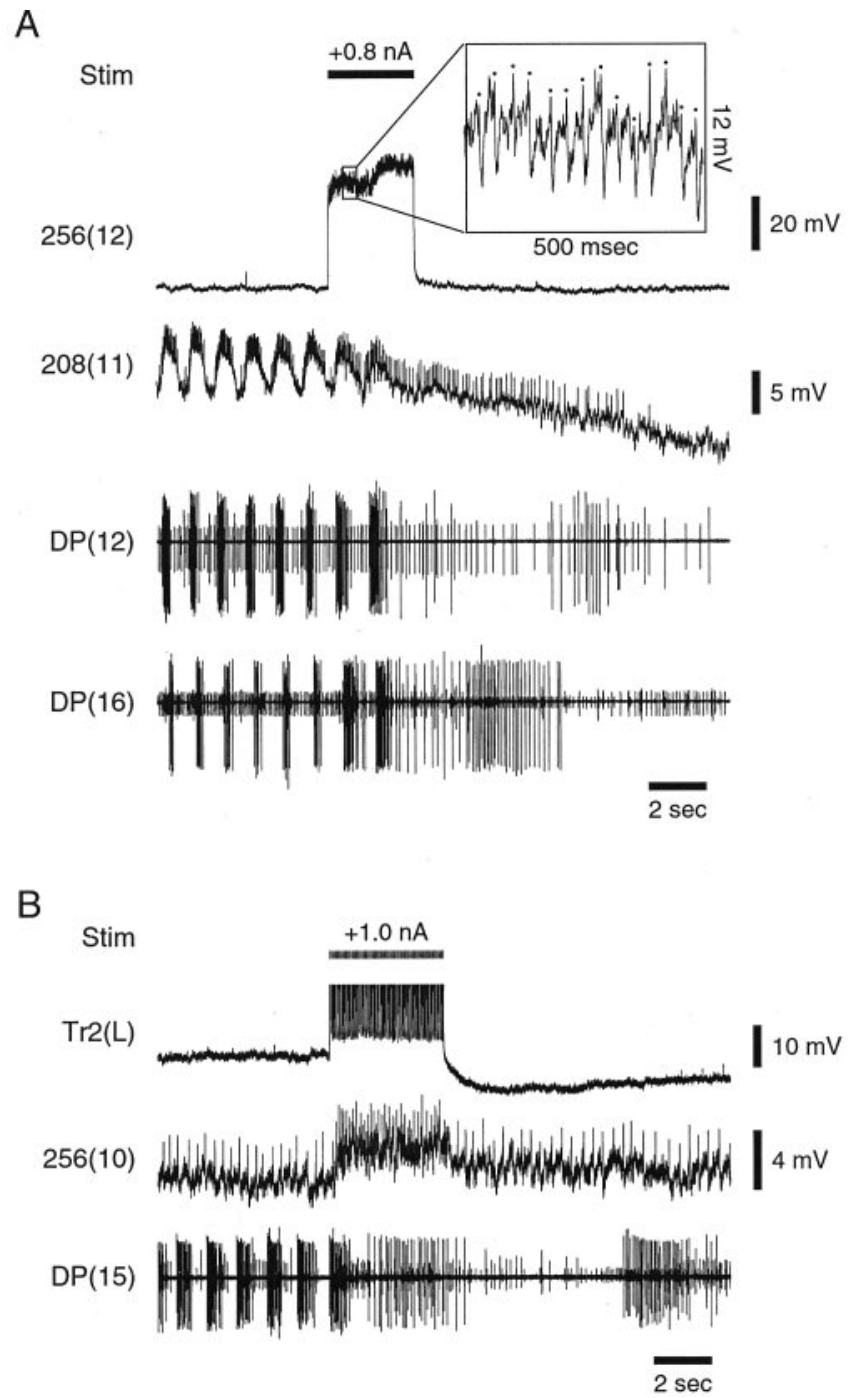

Figure 4. Cell 256 stops swimming. A, Examples of a swim terminated by cell 256 . Simultaneous recordings from cell 256(12), cell 208(11) (a swim (PG cell), and the DP nerves in segments 12 and 16 are shown. Bursts of spikes in the DP nerves at $\sim 1 \mathrm{~Hz}$ indicate an ongoing swim episode (Ort et al., 1974). The bar indicates when current $(+0.8 \mathrm{nA})$ was being passed. The inset shows the spiking activity of cell 256 . Individual spikes are indicated by dots. The average spike rate of cell 256 during current passage was $32 \mathrm{~Hz}$ in this trial. $B$, Cell 256 activity during a cell $\operatorname{Tr} 2$-induced swim termination. Simultaneous recordings from cell $\operatorname{Tr} 2(\mathrm{~L})$, cell 256(10), and the DP nerve in segment 15 during a cell Tr2-induced swim stop are shown. The bar shows when current was passed. Current was delivered in a 4 sec train of $25 \mathrm{msec},+1.0 \mathrm{nA}$ pulses at $20 \mathrm{~Hz}$. The cell $\mathrm{Tr} 2$ recording saturated during current passage and is truncated in the figure. The average spike rate of cell 256(10) during the stimulus was $7.3 \mathrm{~Hz}$.

252 did depolarize when $\operatorname{Tr} 2$ stimulation stopped swimming $(n=1)$, but driving cell 252 during swimming in three animals had weak and variable effects (data not shown).

Finally, none of the identified cell $\operatorname{Tr} 2$ targets seemed to function as either a swim CPG cell or as a swim-initiating cell. None underwent membrane potential oscillations during swimming, and none initiated swimming reliably when stimulated in the quiescent animal (data not shown).

\section{Discussion}

We identified three novel synaptic targets of cell Tr2 using FRET voltage-sensitive dye imaging. Previously, no monosynaptic targets of cell $\operatorname{Tr} 2$ were known. All target neurons were found reliably 


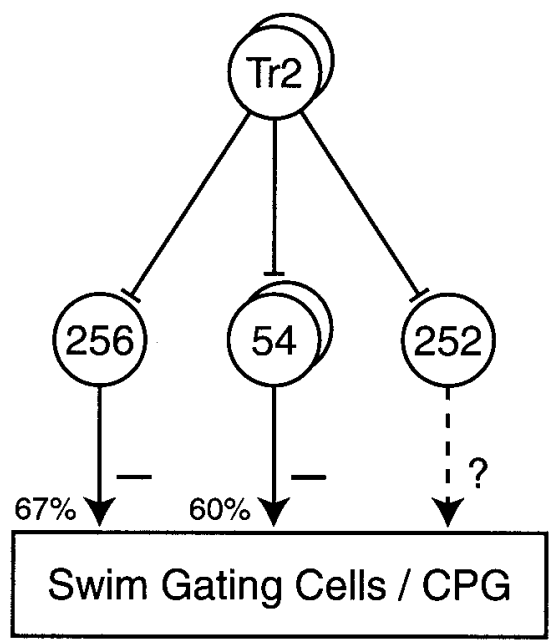

Figure 5. Summary of cell Tr2 targets and their effects on swimming. Connections drawn with bars indicate monosynaptic chemical excitatory connections. Arrows with negative signs next to them indicate an observed inhibitory effect the neuronal basis of which has not been identified. Percentages next to arrows indicate the fraction of animals in which the given cell (or cell pair, for cells 54) was effective in stopping swimming. The dashed arrow with a question mark next to it indicates an unknown effect.

across animals, in multiple midbody ganglia. Two of the targets, cells 256 and 54, stopped swimming when driven to fire at relatively high rates. These results suggest that cells 256 and 54, and possibly cell 252 , provide a segmental neuronal layer that relays the activity of cell $\operatorname{Tr} 2$ to the swim-gating cells or to the swim CPG (Fig. 5). Thus we have identified what is likely to be the first step of the inhibitory neuronal pathway from cell $\operatorname{Tr} 2$ to the swim CPG.

\section{What is the role of cells 256 and 54 in behavior?}

We have shown that stimulating individual cells 256 and 54 can stop swimming but that the rates required are higher than those observed in the cells when driving cell Tr2 to stop swimming (Fig. 4). This is not surprising. Cells 256 and 54 were present in all four segmental ganglia searched, and it is probable that homologs exist in most of the 21 midbody ganglia. When all of these homologs are activated by cell $\operatorname{Tr} 2$ activity, the summed effect is likely to be quite strong. In this light, it is perhaps surprising that stimulating a single cell 256 or cell 54 is able to stop swimming. It seems reasonable that the combined activity of perhaps 20 cells 256 and 40 cells 54, all firing at modest rates, would be sufficient to explain the swim-terminating effects of cell $\operatorname{Tr} 2$.

If the entire functional role of cells 256 and 54 were to relay the activity of cell $\operatorname{Tr} 2$, it would seem simpler for cell $\operatorname{Tr} 2$ to synapse directly onto the swim CPG cells to stop swimming. In Xenopus tadpoles, for instance, reticulospinal neurons that stop an ongoing swim when stimulated connect directly onto a class of spinal motor neurons that help generate the swim rhythm (Roberts et al., 1997; Perrins et al., 2002). What does having cells 256 and 54 in the circuit contribute that could not be achieved by having cell $\operatorname{Tr} 2$ synapse directly onto the swim-gating cells or the swim CPG cells?

One possibility is that cells 256 and 54 act to distribute the output of cell $\operatorname{Tr} 2$. That is, each cell $\operatorname{Tr} 2$ could synapse on some $20-40$ cells 256 or 54 , or both, and each of these could then synapse on some number of swim CPG cells, call it $n$. In this way, the activity of cell $\operatorname{Tr} 2$ would effect $20 n-40 n$ downstream cells, although making only 20-40 synapses itself. This might be met- abolically more efficient than having cell $\mathrm{Tr} 2$ make synapses onto all 20n-40n downstream cells and would effectively be a form of amplification.

\section{Integration of anti-swimming inputs}

Another possibility is that cells 256 and 54 have a broader role in the leech nervous system. It seems likely that their activity is not simply a function of the activity of cell $\operatorname{Tr} 2$ but reflects inputs from other neurons as well. For instance, cells 256 and 54 might act as integrators of various anti-swimming inputs. Previous authors have shown that there are parallel swim-activating and swim-inactivating systems in the leech (Brodfuehrer and Burns, 1995), and it is possible that cells 256 and 54 serve as part of an anti-swim-gating network, parallel to the swim-gating cells (cells 204, 21, and 61). These two networks would be expected to inhibit one another, thus forming a distributed "decision system" for swimming. Various pro-swimming and anti-swimming inputs would converge on the swim-gating and anti-swim-gating systems, and whichever system received the greater net input would prevail. Such a scheme has been proposed for neurons in the medial temporal and lateral intraparietal areas of monkey cortex that are thought to mediate judgments about motion directionality (Shadlen and Newsome, 1996, 2001; Shadlen et al., 1996; Gold and Shadlen, 2000).

Some support for such an anti-swim-gating hypothesis comes from the observation that the organization of the cell Tr2 swimterminating circuitry seems to grossly parallel elements of the swim-initiation circuitry. In part of the swim-initiation circuitry, cell $\operatorname{Tr} 1$, a head brain cell pair, synapses on segmental swimgating cells. These cells then connect to the swim CPG (Brodfuehrer and Friesen, 1986a,b; Nusbaum and Kristan, 1986). Similarly, cell Tr2, a head brain cell pair, synapses on cells 256 and 54, which then (directly or indirectly) connect to the swim CPG. In both systems, however, it seems that the connections of the segmental cells are not strictly intrasegmental: cell 204 makes all its known connections with CPG cells intersegmentally (although intrasegmental connections may exist), and the morphology of cells 256 and 54 suggests that they too are likely to make many of their synapses intersegmentally. Thus the organization of the cell Tr2 swim-terminating circuitry seems roughly to parallel the cell Tr1 circuitry for swim initiation.

\section{Local control}

In the anti-swim-gating scheme described above, all of the cells 256 and 54 in the animal are collectively "representing" a single thing: the extent to which the current situation of the animal favors or disfavors swimming. Another possibility is that the cells 256 and 54 in different segments are representing different things. For instance, cell 256 or 54 (or both) might play the role of a "segmental swim slowing" neuron, which is used by the leech CNS to slow down or phase delay the swim oscillation locally (in the same segment and perhaps a few neighboring segments). This might be useful as part of a sensory feedback loop acting to maintain the proper phase in each segment in the face of local perturbations. Such local feedback, although not mediated by non-CPG interneurons, has been identified in the leech and lamprey swim circuits (Viana Di Prisco et al., 1990; Wallen, 1997; Cang and Friesen, 2000; Cang et al., 2001). If cells 256 and 54 play such a role, it would presumably complement the direct feedback identified previously (Cang et al., 2001). 
A similar possibility is that cell 256 or 54 plays the role of a "segmental shortening" neuron. Such a neuron could be used during crawling to generate the coordinated wave of local shortening that comprises the contraction phase of a crawl step. Such cells have been posited to exist in a model of the leech crawl CPG (Cacciatore et al., 2000). In both this and the segmental-swimslowing scheme, cells 256 and 54 would effectively serve two related but distinct roles: to mediate swim termination by $\operatorname{Tr} 2$ and coordinate other behaviors.

\section{Using imaging to find synaptic targets}

We found imaging to be an essential tool in identifying synaptic targets of cell Tr2. Even having established the morphology and electrophysiological properties of cell 256, we relied on imaging to find this cell in each preparation. The exact position of the soma of cell 256 varies and is in an area of the ganglion with many somata of similar size. Therefore, our primary means of identifying the cell is to drive cell Tr2 and image all the neurons in its general location. In contrast, we can reliably identify cells 252 and 54 solely on the basis of soma position and spike shape, although we used imaging for their initial discovery.

It should be noted that other targets of cell Tr2 may exist. Although the optical method greatly facilitates the search for synaptic targets, it does not guarantee that one will find all of them. In particular, the dorsal side of the midbody ganglia was not examined as extensively as the ventral side, so there may be additional $\operatorname{Tr} 2$ targets with their somata on the dorsal surface.

Our work is related to previous work using imaging to explore connectivity. Kozloski et al. (2001) recently used calcium imaging to explore cortical connectivity in the mouse, building on earlier work developing the technique (Peterlin et al., 2000). Those authors used imaging to find neurons in mouse cortex that are targets of an impaled neuron. This enabled them to examine the cell-type specificity of cortical microcircuitry. The ability to record the electrical activity of a number of individual neurons, using various optical techniques, should open the way for establishing the connectivity of neuronal circuits in both simple and complex brains.

\section{References}

Berry MS, Pentreath VW (1976) Criteria for distinguishing between monosynaptic and polysynaptic transmission. Brain Res 105:1-20.

Brodfuehrer PD, Burns A (1995) Neuronal factors influencing the decision to swim in the medicinal leech. Neurobiol Learn Mem 63:192-199.

Brodfuehrer PD, Friesen WO (1986a) Initiation of swimming activity by trigger neurons in the leech subesophageal ganglion. I. Output connections of Tr1 and Tr2. J Comp Physiol [A] 159:489-502.

Brodfuehrer PD, Friesen WO (1986b) Initiation of swimming activity by trigger neurons in the leech subesophageal ganglion. II. Role of segmental swim-initiating interneurons. J Comp Physiol [A] 159:503-510.

Byrne JH, Castellucci VF, Kandel ER (1978) Contribution of individual mechanoreceptor sensory neurons to defensive gill-withdrawal reflex in Aplysia. J Neurophysiol 41:418-431.

Cacciatore TW, Brodfuehrer PD, González JE, Jiang T, Adams SR, Tsien RY, Kristan Jr WB, Kleinfeld D (1999) Identification of neural circuits by imaging coherent electrical activity with FRET-based dyes. Neuron 23:449-459.

Cacciatore TW, Rozenshteyn R, Kristan WB Jr (2000) Kinematics and modeling of leech crawling: evidence for an oscillatory behavior produced by propagating waves of excitation. J Neurosci 20:1643-1655.

Cang J, Friesen WO (2000) Sensory modification of leech swimming: rhythmic activity of ventral stretch receptors can change intersegmental phase relationships. J Neurosci 20:7822-7829.

Cang J, Yu X, Friesen WO (2001) Sensory modification of leech swimming: interactions between ventral stretch receptors and swim-related neurons. J Comp Physiol [A] 187:569-579.

Frost WN, Katz PS (1996) Single neuron control over a complex motor pattern. Proc Natl Acad Sci USA 93:422-426.

Gold JI, Shadlen MN (2000) Representation of a perceptual decision in developing oculomotor commands. Nature 404:390-394.

González JE, Tsien RY (1995) Voltage sensing by fluorescence resonance energy transfer in single cells. Biophys J 69:1272-1280.

González JE, Tsien RY (1997) Improved indicators of cell membrane potential that use fluorescence resonance energy transfer. Chem Biol 4:269-277.

González JE, Oades K, Leychkis Y, Harootunian A, Negulescu PA (1999) Cell-based assays and instrumentation for screening ion-channel targets. Drug Discov Today 4:431-439.

Grillner S, Georgopoulos AP, Jordan LM (1997) Selection and initiation of motor behavior. In: Neurons, networks, and motor behavior (Stein PSG, Grillner S, Selverston AI, Stuart DG, eds), pp 3-19. Cambridge, MA: MIT. Hannan EJ (1970) Multiple time series. New York: Wiley.

Jarvis MR, Mitra PP (2001) Sampling properties of the spectrum and coherency of sequences of action potentials. Neural Comput 13:717-749.

Kozloski J, Hamzei-Sichani F, Yuste R (2001) Stereotyped position of local synaptic targets in neocortex. Science 293:868-872.

Kristan Jr WB, Shaw BK (1997) Population coding and behavioral choice. Curr Opin Neurobiol 7:826-831.

Kupfermann I, Weiss KR (1978) The command neuron concept. Behav Brain Sci 1:3-39.

Liao X, Walters ET (2002) The use of elevated divalent cation solutions to isolate monosynaptic components of sensorimotor connections in Aplysia. J Neurosci Methods 120:45-54.

Muller KJ, Nicholls JG, Stent GS (1981) Neurobiology of the leech. Cold Spring Harbor, NY: Cold Spring Harbor Laboratory.

Nicholls JG, Purves D (1970) Monosynaptic chemical and electrical connexions between sensory and motor cells in the central nervous system of the leech. J Physiol (Lond) 209:647-667.

Nusbaum MP, Kristan Jr WB (1986) Swim initiation in the leech by serotonin-containing interneurones, cells 21 and 61. J Exp Biol 122:277-302.

O'Gara BA, Friesen WO (1995) Termination of leech swimming activity by a previously identified swim trigger neuron. J Comp Physiol [A] 177:627-636

Orlovsky GN, Deliagina TG, Grillner S (1999) Neuronal control of locomotion: from mollusc to man. Oxford: Oxford UP.

Ort CA, Kristan Jr WB, Stent GS (1974) Neuronal control of swimming in the medicinal leech. II. Identification and connections of motor neurons. J Comp Physiol [A] 94:121-154.

Pearson KG (1993) Common principles of motor control in vertebrates and invertebrates. Annu Rev Neurosci 16:265-297.

Percival DB, Walden AT (1993) Spectral analysis for physical applications. Cambridge, UK: Cambridge UP.

Perrins R, Walford A, Roberts A (2002) Sensory activation and role of inhibitory reticulospinal neurons that stop swimming in hatchling frog tadpoles. J Neurosci 22:4229-4240.

Peterlin ZA, Kozloski J, Mao BQ, Tsiola A, Yuste R (2000) Optical probing of neuronal circuits with calcium indicators. Proc Natl Acad Sci USA 97:3619-3624.

Rink TJ, Montecucco C, Hesketh TR, Tsien RY (1980) Lymphocyte membrane potential assessed with fluorescent probes. Biochim Biophys Acta 595:15-30.

Roberts A, Soffe SR, Perrins R (1997) Spinal networks controlling swimming in hatchling Xenopus tadpoles. In: Neurons, networks, and motor behavior (Stein PSG, Grillner S, Selverston AI, Stuart DG, eds), pp 83-90. Cambridge, MA: MIT.

Shadlen MN, Newsome WT (1996) Motion perception: seeing and deciding. Proc Natl Acad Sci USA 93:628-633.

Shadlen MN, Newsome WT (2001) Neural basis of a perceptual decision in the parietal cortex (area LIP) of the rhesus monkey. J Neurophysiol 86:1916-1936

Shadlen MN, Britten KH, Newsome WT, Movshon JA (1996) A computational analysis of the relationship between neuronal and behavioral responses to visual motion. J Neurosci 16:1486-1510. 
Thomson DJ (1982) Spectral estimation and harmonic analysis. Proc IEEE 70:1055-1096.

Thomson DJ, Chave AD (1991) Jackknifed error estimates for spectra, coherences, and transfer functions. In: Advances in spectrum analysis and array processing (Shykin S, ed), pp 58-113. Englewood Cliffs, NJ: Prentice Hall.

Tsien RY (1976) The design and use of organic chemical tools in cellular physiology. $\mathrm{PhD}$ thesis, University of Cambridge.

Viana Di Prisco G, Wallen P, Grillner S (1990) Synaptic effects of intraspinal stretch receptor neurons mediating movement-related feedback during locomotion. Brain Res 530:161-166.

Wallen P (1997) Spinal networks and sensory feedback in the control of undulatory swimming in lamprey. In: Neurons, networks, and motor behavior (Stein PSG, Grillner S, Selverston AI, Stuart DG, eds), pp 75-82. Cambridge, MA: MIT.

Weeks JC (1981) Neuronal basis of leech swimming: separation of swim initiation, pattern generation, and intersegmental coordination by selective lesions. J Neurophysiol 45:698-723.

Weeks JC, Kristan Jr WB (1978) Initiation, maintenance, and modulation of swimming in the medicinal leech by the activity of a single neuron. J Exp Biol 77:71-88.

Zochowski M, Wachowiak M, Falk CX, Cohen LB, Lam YW, Antic S, Zecevic D (2000) Imaging membrane potential with voltage-sensitive dyes. Biol Bull 198:1-21. .0 